\title{
Higgs pair production with SUSY QCD correction: revisited under current experimental constraints
}

\author{
Chengcheng Han, ${ }^{a}$ Xuanting $\mathrm{Ji}^{a, b}$ Lei Wu, ${ }^{c}$ Peiwen $\mathbf{W u}^{a}$ and Jin Min Yang ${ }^{a}$ \\ ${ }^{a}$ State Key Laboratory of Theoretical Physics, Institute of Theoretical Physics, Academia Sinica, \\ Beijing 100190, China \\ ${ }^{b}$ Institute of Theoretical Physics, College of Applied Science, Beijing University of Technology, \\ Beijing 100124, China \\ ${ }^{c}$ ARC Centre of Excellence for Particle Physics at the Terascale, School of Physics, \\ University of Sydney, \\ Sydney, NSW 2006, Australia \\ E-mail: hancheng@itp.ac.cn, xuantingji@gmail.com, \\ leiwu@physics.usyd.edu.au, pwwu@itp.ac.cn, jmyang@itp.ac.cn
}

ABSTRACT: We consider the current experimental constraints on the parameter space of the MSSM and NMSSM. Then in the allowed parameter space we examine the Higgs pair production at the $14 \mathrm{TeV}$ LHC via $b \bar{b} \rightarrow h h$ ( $h$ is the $125 \mathrm{GeV}$ SM-like Higg boson) with one-loop SUSY QCD correction and compare it with the production via $g g \rightarrow h h$. We obtain the following observations: (i) For the MSSM the production rate of $b \bar{b} \rightarrow h h$ can reach $50 \mathrm{fb}$ and thus can be competitive with $g g \rightarrow h h$, while for the NMSSM $b \bar{b} \rightarrow h h$ has a much smaller rate than $g g \rightarrow h h$ due to the suppression of the $h b \bar{b}$ coupling; (ii) The SUSY-QCD correction to $b \bar{b} \rightarrow h h$ is sizable, which can reach $45 \%$ for the MSSM and $15 \%$ for the NMSSM within the $1 \sigma$ region of the Higgs data; (iii) In the heavy SUSY limit (all soft mass parameters become heavy), the SUSY effects decouple rather slowly from the Higgs pair production (especially the $g g \rightarrow h h$ process), which, for $M_{\mathrm{SUSY}}=5 \mathrm{TeV}$ and $m_{A}<1 \mathrm{TeV}$, can enhance the production rate by a factor of 1.5 and 1.3 for the MSSM and NMSSM, respectively. So, the Higgs pair production may be helpful for unraveling the effects of heavy SUSY.

KEYWORDS: Supersymmetry Phenomenology, NLO Computations

ArXiv EPrint: 1307.3790 


\section{Contents}

1 Introduction 1

2 A description of models and analytic calculations $\quad 2$

3 Numerical studies $\quad 4$

3.1 A scan of parameter space 4

3.2 The cross section of $b \bar{b} \rightarrow h h$ with SUSY-QCD correction 6

4 Conclusion

\section{Introduction}

The discovery of a Higgs boson at around $125 \mathrm{GeV}$ has been announced by the ATLAS and CMS collaborations $[1,2]$. Up to now, the measurements of the Higgs boson properties are in good agreement with the Standard Model (SM) predictions except for the enhanced diphoton rate $\sigma / \sigma_{\mathrm{SM}}=1.65_{-0.30}^{+0.34}$ reported by the ATLAS collaboration. The future precise measurements will further test the SM and allow for a probe for new physics like supersymmetry (SUSY) which is a promising framework to accommodate such a $125 \mathrm{GeV}$ Higgs boson [3-13]. Therefore, the intensive studies of the Higgs productions and decays are very important and urgent.

Among the productions of the Higgs boson at the LHC, the pair production is a rare process but quite important since it can be used to measure the Higgs self-couplings [14]. On the experimental side, the discovery potential of Higgs pair signal at the LHC has been studied by analyzing the decay channels $h h \rightarrow b \bar{b} \gamma \gamma / b \bar{b} \mu^{+} \mu^{-}$[15]. Recently, the jet substructure technique was applied to the Higgs pair production in the boosted final states [16], such as $h h \rightarrow b \bar{b} \tau^{+} \tau^{-} / b \bar{b} W^{+} W^{-}$[17-20], which was found to be powerful in observing the events at the $14 \mathrm{TeV}$ LHC with $600 \mathrm{fb}^{-1}$ integrated luminosity [20]. On the theoretical side, in the SM the main pair production mechanism is found to be the gluon fusion $g g \rightarrow h h$ via heavy quark loops [21-26]. Numerous studies have also been performed for Higgs pair production in new physics models [27-36]. Note that although the bottom quark annilation $b \bar{b} \rightarrow h h$ has a much smaller rate than the gluon fusion process in the SM [37-39], it can be significantly enhanced via the enlarged $h b \bar{b}$ coupling in new physics models like the Minimal Supersymmetric Standard Model (MSSM) [40].

In this work, we revisit the Higgs pair production in SUSY for two reasons. One is that the sizable SUSY-QCD correction must be considered for $b \bar{b} \rightarrow h h$, which has been presented in the MSSM but not in the NMSSM [41, 42]. The other is that the studies should be updated by using the latest experimental constraints including the recent LHC Higgs data, the LHCb $B_{s} \rightarrow \mu^{+} \mu^{-}$data and the Planck dark matter relic density. It is 
also notable that the masses of the third generation sparticles involved in the SUSY-QCD correction to $b \bar{b} \rightarrow h h$ have been pushed up to a few hundred GeV by the LHC direct searches [43]. So the size of such a correction will be quite different from the previous results in the literature $[40,44]$.

This paper is organized as follows. In section 2 we briefly review the Higgs sectors in the MSSM and NMSSM and give a description of the analytic calculation of the SUSY-QCD correction. Then in section 3 we present the numerical results of Higgs pair production at the LHC and discuss the SUSY-QCD residual effects in the heavy sparticle limit. Finally, we draw the conclusion in section 4 .

\section{A description of models and analytic calculations}

In the MSSM there are two complex Higgs doublets, $H_{u}$ and $H_{d}$, which give rise to five physical Higgs bosons: two CP-even $(h, H)$, one CP-odd $(A)$ and a charged pair $\left(H^{ \pm}\right)$. Due to the $\mu$ term appearing in the superpotential, the MSSM suffers from the $\mu$-problem. Besides, in order to give a $125 \mathrm{GeV}$ SM-like Higgs boson, large corrections to the Higgs mass from heavy stops is needed, which will lead to the little fine tuning problem. To overcome these difficulties, we can go beyond the MSSM. One alternative is the NMSSM, which introduces a singlet Higgs field. In the NMSSM the $\mu$ term does not appear in the superpotential. Instead, it is generated when the singlet Higgs field develops a vev. Also, the SM-like Higgs boson gets an extra tree-level mass from the mixing with the singlet field and thus the stops are not necessarily heavy to push up the Higgs mass, which alleviates the little fine-tuning problem [45-52]. In the NMSSM the singlet Higgs field mixes with the other two doublet scalars. Then the Higgs sector contains seven Higgs bosons, i.e., compared with the five Higgs bosons in the MSSM, the NMSSM contains one more CPeven and one more CP-odd Higgs bosons. In the following $H_{1,2}$ denote the real scalar components of $H_{d, u}$ in the MSSM and $H_{1,2,3}$ denote the real scalar components of $H_{d, u, s}$ in the NMSSM. $\tan \beta \equiv v_{u} / v_{d}$ is also used in our paper (here $H_{d}, H_{u}$ and $H_{s}$ are the downtype, up-type and singlet Higgs fields, respectively). One can get the mass eigenstates from the CP-even states:

$$
\begin{array}{rll}
\text { MSSM : } & h_{i}=U_{i j} H_{j} & (i, j=1,2), \\
\text { NMSSM : } & h_{i}=V_{i j} H_{j} & (i, j=1,2,3)
\end{array}
$$

where $U_{i 1}^{2}+U_{i 2}^{2}=1, V_{i 1}^{2}+V_{i 2}^{2}+V_{i 3}^{2}=1$ and the $h_{i}$ is aligned by mass. The singlet contribution is reflected by the rotation matrix elements $V_{i 3}$ via the formula $h_{\mathrm{SM}}=$ $V_{h_{\mathrm{SM}} 1} H_{1}+V_{h_{\mathrm{SM}} 2} H_{2}+V_{h_{\mathrm{SM}} 3} H_{3}$ (a large $V_{h_{\mathrm{SM}} 3}$ means that $h_{\mathrm{SM}}$ has a considerable singlet component).

In our calculations, we follow the simplified ACOT prescription to deal with the $b$ quark mass [55-57]. By including the QCD and SUSY-QCD effects to the bottom Yukawa couplings, we can respectively obtain the effective $h_{i} b \bar{b}$ couplings in the MSSM [58-66] and 
NMSSM [67]:

$$
\begin{aligned}
\text { MSSM : } & y_{h_{i} b b} \rightarrow \frac{g m_{b}^{\overline{D R}}}{2 M_{W}} \frac{U_{i 1}}{\cos \beta} \Delta_{b i}^{\mathrm{MSSM}} & (i=1,2), \\
\text { NMSSM : } & y_{h_{i} b b} \rightarrow \frac{g m_{b}^{\overline{D R}}}{2 M_{W}} \frac{V_{i 1}}{\cos \beta} \Delta_{b i}^{\mathrm{NMSSM}} & (i=1,2,3)
\end{aligned}
$$

where

$$
\begin{aligned}
\Delta_{b i}^{\mathrm{MSSM}} & =\frac{1}{1+\Delta_{b}^{1}}\left(1+\Delta_{b}^{1} \frac{U_{i 2}}{U_{i 1} \tan \beta}\right)(i=1,2), \\
\Delta_{b i}^{\mathrm{NMSSM}} & =\frac{1}{1+\Delta_{b}^{1}}\left[1+\Delta_{b}^{1}\left(\frac{V_{i 2}}{V_{i 1} \tan \beta}+\frac{V_{i 3} v_{d}}{V_{i 1} v_{s}}\right)\right](i=1,2,3), \\
\Delta_{b} & =\frac{2 \alpha_{s}}{3 \pi} m_{\tilde{g}} \mu \tan \beta I\left(m_{\tilde{b}_{1}}^{2}, m_{\tilde{b}_{2}}^{2}, m_{\tilde{g}}^{2}\right) \\
\Delta_{b}^{2} & =-\frac{2 \alpha_{s}}{3 \pi} m_{\tilde{g}} A_{b} I\left(m_{\tilde{b}_{1}}^{2}, m_{\tilde{b}_{2}}^{2}, m_{\tilde{g}}^{2}\right), \Delta_{b}^{1}=\frac{\Delta_{b}}{1+\Delta_{b}^{2}}
\end{aligned}
$$

Here it should be noted that due to the contribution of the singlet field to the effective potential, an additional correction term $\Delta_{b}^{1} \frac{V_{i 3} v_{d}}{V_{i 1} v_{s}}$ appears in the NMSSM. The $v_{d}$ and $v_{s}$ are the VEVs of the Higgs fields $H_{u}$ and $H_{d}$ respectively. The auxiliary function $I$ is defined as

$$
I(a, b, c)=-\frac{1}{(a-b)(b-c)(c-a)}\left(a b \ln \frac{a}{b}+b c \ln \frac{b}{c}+c a \ln \frac{c}{a}\right) .
$$

The value of $m_{b}^{\overline{D R}}$ is related to the QCD- $\overline{\mathrm{MS}}$ mass $m_{b}^{\overline{\mathrm{MS}}}$ (which is usually taken as an input parameter [68]) by

$$
m_{b}^{\overline{D R}}\left(\mu_{R}\right)=m_{b}^{\overline{M S}}\left(\mu_{R}\right)\left[1-\frac{\alpha_{s}}{3 \pi}-\frac{\alpha_{s}^{2}}{144 \pi^{2}}(73-3 n)\right]
$$

where $n$ is the number of active quark flavors and $m_{b}^{\overline{M S}}\left(\mu_{R}\right)$ is taken as

$$
m_{b}^{\overline{\mathrm{MS}}}\left(\mu_{R}\right)= \begin{cases}U_{6}\left(\mu_{R}, m_{t}\right) U_{5}\left(m_{t}, \bar{m}_{b}\right) \bar{m}_{b}\left(\bar{m}_{b}\right) & \text { for } \quad \mu_{R}>m_{t} \\ U_{5}\left(\mu_{R}, \bar{m}_{b}\right) \bar{m}_{b}\left(\bar{m}_{b}\right) & \text { for } \quad \mu_{R} \leq m_{t} .\end{cases}
$$

When $Q_{2}>Q_{1}$, the evolution factor $U_{n}$ reads

$$
U_{n}\left(Q_{2}, Q_{1}\right)=\left(\frac{\alpha_{s}\left(Q_{2}\right)}{\alpha_{s}\left(Q_{1}\right)}\right)^{d_{n}}\left[1+\frac{\alpha_{s}\left(Q_{1}\right)-\alpha_{s}\left(Q_{2}\right)}{4 \pi} J_{n}\right]
$$

where

$$
d_{n}=\frac{12}{33-2 n}, \quad J_{n}=-\frac{8982-504 n+40 n^{2}}{3(33-2 n)^{2}}
$$

Since the $\Delta_{b}$-related corrections have already been included into the tree-level contribution, we need the following counter terms to subtract them to avoid double counting in 
the one-loop calculations [58]

$$
\begin{array}{rlr}
\text { MSSM : } & \delta \tilde{m}_{b}^{h_{i}}=m_{b}^{\overline{D R}}\left(1-\frac{U_{i 2}}{U_{i 1} \tan \beta}\right) \Delta_{b}^{1}, & (i=1,2), \\
\text { NMSSM : } & \delta \tilde{m}_{b}^{h_{i}}=m_{b}^{\overline{D R}}\left(1-\frac{V_{i 2}}{V_{i 1} \tan \beta}-\frac{V_{i 3} v_{d}}{V_{i 1} v_{s}}\right) \Delta_{b}^{1}, & (i=1,2,3) .
\end{array}
$$

For SUSY-QCD corrections to $b \bar{b} \rightarrow h h$, the sbottoms and gluino are involved in the loops. The sbottom mass matrix is given by [69]

$$
M_{\tilde{b}}^{2}=\left(\begin{array}{cc}
m_{\tilde{b}_{L}}^{2} & m_{b} X_{b}^{\dagger} \\
m_{b} X_{b} & m_{\tilde{b}_{R}}^{2}
\end{array}\right),
$$

where

$$
\begin{aligned}
m_{\tilde{b}_{L}}^{2} & =m_{\tilde{Q}}^{2}+m_{b}^{2}-m_{Z}^{2}\left(\frac{1}{2}-\frac{1}{3} \sin ^{2} \theta_{W}\right) \cos (2 \beta), \\
m_{\tilde{b}_{R}}^{2} & =m_{\tilde{D}}^{2}+m_{b}^{2}-\frac{1}{3} m_{Z}^{2} \sin ^{2} \theta_{W} \cos (2 \beta), \\
X_{b} & =A_{b}-\mu \tan \beta .
\end{aligned}
$$

After diagonalizing eq. (2.13), we can obtain the sbottom masses $m_{\tilde{b}_{1,2}}$ and the mixing angle $\theta_{\tilde{b}}$ :

$$
\begin{aligned}
m_{\tilde{b}_{1,2}} & =\frac{1}{2}\left[m_{\tilde{b}_{L}}^{2}+m_{\tilde{b}_{R}}^{2} \mp \sqrt{\left(m_{\tilde{b}_{L}}^{2}-m_{\tilde{b}_{R}}^{2}\right)^{2}+4 m_{b}^{2} X_{b}^{2}}\right], \\
\tan 2 \theta_{\tilde{b}} & =\frac{2 m_{b} X_{b}}{m_{\tilde{b}_{L}}^{2}-m_{\tilde{b}_{R}}^{2}} .
\end{aligned}
$$

The Feynman diagrams for one-loop SUSY-QCD corrections to $b \bar{b} \rightarrow h h$ has been represented in [44]. To preserve supersymmetry, we adopt the dimension reduction method to regulate the UV divergences in the gluino and squark loops. Then we use the on-shell renormalization scheme to remove these UV divergences.

\section{$3 \quad$ Numerical studies}

\subsection{A scan of parameter space}

We use NMSSMTools [70-72] and LoopTools [73] to perform a random scan over the parameter space and loop calculations. For simplicity, we assume an universal parameter $M_{L 3}$ for the slepton sector and fix all irrelevant soft parameters for first two generation of the squark sector to be $1 \mathrm{TeV}$. We also set $M_{D 3}=M_{U 3}$ and $A_{b}=A_{t}$ for the third generation of the squarks. Besides, we impose the grand unification relation of the gaugino masses, $3 M_{1} / 5 \alpha_{1}=M_{2} / \alpha_{2}=M_{3} / \alpha_{3}$, and treat $M_{1}$ as an input parameter. The parameter ranges in our scan are: 
(a) For the MSSM

$$
\begin{aligned}
1 & \leq \tan \beta \leq 60,100 \mathrm{GeV} \leq M_{A} \leq 1 \mathrm{TeV}, 100 \mathrm{GeV} \leq \mu \leq 2 \mathrm{TeV} \\
100 \mathrm{GeV} & \leq M_{Q 3}, M_{U 3} \leq 2 \mathrm{TeV}, 100 \mathrm{GeV} \leq M_{L 3} \leq 1 \mathrm{TeV} \\
\left|A_{t}\right| & \leq 5 \mathrm{TeV}, 50 \mathrm{GeV} \leq M_{1} \leq 500 \mathrm{GeV} .
\end{aligned}
$$

(b) For the NMSSM

$$
\begin{aligned}
0.5 & \leq \lambda \leq 0.7,0.1 \leq \kappa \leq 0.51,\left|A_{\kappa}\right| \leq 1 \mathrm{TeV} \\
1 & \leq \tan \beta \leq 10,100 \mathrm{GeV} \leq \mu \leq 600 \mathrm{GeV}, 100 \mathrm{GeV} \leq M_{A} \leq 1 \mathrm{TeV} \\
100 \mathrm{GeV} & \leq M_{Q 3}, M_{U 3} \leq 2 \mathrm{TeV}, 100 \mathrm{GeV} \leq M_{L 3} \leq 1 \mathrm{TeV} \\
\left|A_{t}\right| & \leq 5 \mathrm{TeV}, 50 \mathrm{GeV} \leq M_{1} \leq 500 \mathrm{GeV} .
\end{aligned}
$$

In our scan we consider the following experimental constraints:

(i) The bounds for Higgs boson from the LEP, Tevatron and LHC experiments and require the SM-like Higgs mass to be in the range of $123 \mathrm{GeV}<m_{h}<127 \mathrm{GeV}$; Here we require the surviving samples to explain the observable at $2 \sigma$ level which has an experimental central value. For the LEP and Tevatron limits, the upper or lower bounds are implemented in our scan. For the LHC Higgs search of $H / A \rightarrow \tau \tau$ [74] and $H^{ \pm} \rightarrow \tau \nu_{\tau}$ [75], we require the samples to satisfy the upper limits.

(ii) The constraints from the precision electroweak data $[76,77]$ and flavor physics at $2 \sigma$ level;

(iii) The dark matter relic density from Plank at $3 \sigma$ level and the limit of direct detection from XENON100 [78];

(iv) The explanation of muon $g-2$ at $2 \sigma$ level [79].

In our scan, for each experimental data which has a central value, we require the samples to agree with the experimental data at $2 \sigma$ level, except for the dark matter relic density which is required to agree with the measured value at $3 \sigma$ level (we made such a choice just in order to be consistent with the analysis in the literature). For the LEP and Tevatron direct search bounds on sparticle masses, we just require the samples to satisfy such bounds. For the LHC Higgs search of $H / A \rightarrow \tau \tau$ and $H^{ \pm} \rightarrow \tau \nu_{\tau}$, we require the samples to satisfy the upper limits. The scan ranges of the parameters are large, we keep the samples survived various experimental constraints as stated above. Besides, we further require gluino mass larger than $1 \mathrm{TeV}$ to avoid multi-jets search on SUSY [80-82]. However, we did not impose other LHC direct limits on sparticles for the following reasons. First, we required the first and second generations of squarks to be $1 \mathrm{TeV}$ and the gluino beyond $1 \mathrm{TeV}$. But the latest LHC search results gave more stringent constraints on such squark and gluino mass (the most stringent bound is for the CMSSM, which is $m_{\tilde{g}}>1.7 \mathrm{TeV}$ in case of $m_{\tilde{g}} \simeq m_{\tilde{q}}$ and $m_{\tilde{g}}>1.1 \mathrm{TeV}$ in case of $\left.m_{\tilde{q}} \gg m_{\tilde{g}}\right)$. Actually, our results are not sensitive to these masses. Second, the current LHC limit is about $500-600 \mathrm{GeV}$ 
for stop and 400-600 GeV for sbottom [83-88]. However, such limits were obtained in some simplified model or by assuming a certain decay branching ratio to be $100 \%$. In our case the stop and sbottom decays are quite complicated, which will weaken the LHC limits. Further, for electroweak gauginos and sleptons, the current LHC limits will also be weakened in our case for the same reason. After that we also require surviving samples to avoid Landau singularity at GUT scale and we checked that all of our surviving samples satisfy $\sqrt{\lambda^{2}+\kappa^{2}}<0.75$ in NMSSM. We note that a large $\tan \beta$ exist in the surviving samples of the MSSM, this is because that a $125 \mathrm{GeV}$ neutral Higgs mass is guaranteed by a large $A_{t}$ (which provides $X_{t} / M_{s}$ close to $\sqrt{6}$ ) even for $\tan \beta$ as large as 40 . As for the flavor constraints, we projected our samples onto the $\tan \beta$ versus the charged Higgs mass plane and found that when $\tan \beta$ increases the charged Higgs mass grows dramatically (especially, for $\tan \beta$ close to 40, the charged Higgs mass is heavier than $700 \mathrm{GeV}$ ) and thus can satisfy the flavor constraints. For the samples surviving the above constraints (i)-(iv), we further perform a fit by using the available Higgs data at the LHC. We define the Higgs signal strength $\mu_{i}$ as

$$
\mu_{i}=\frac{\Sigma_{p} \sigma_{p} \epsilon_{p}^{i}}{\Sigma_{p} \sigma_{p}^{\mathrm{SM}} \epsilon_{p}^{i}} \frac{\mathrm{Br}_{i}}{\mathrm{Br}_{i}^{\mathrm{SM}}}
$$

where $p$ is the Higgs boson production mode and $i$ stands for the measured channels by Tevatron, ATLAS and CMS collaborations. For each production mode $p$, its contribution to the channel $i$ can be determined by the selection efficiency $\epsilon_{p}^{i}$ [89]. We summarize all experimental signal strength $\mu_{i}^{\text {exp }}$ with their $1 \sigma$ error-bars and selection efficiencies in figure 1. We can see that most measurement results are consistent with the SM predictions. The CMS and ATLAS collaborations also reported their observations of the Higgs mass $M_{h}^{\exp }[90,91]$ :

$$
M_{h}^{\exp }=\left\{\begin{array}{l}
125.8 \pm 0.5 \pm 0.2 \mathrm{GeV}(\operatorname{CMS} Z Z) \\
125.4 \pm 0.5 \pm 0.6 \mathrm{GeV}(\operatorname{CMS} \gamma \gamma) \\
124.3 \pm 0.6 \pm 0.5 \mathrm{GeV}(\operatorname{ATLAS} Z Z) \\
126.8 \pm 0.2 \pm 0.7 \mathrm{GeV}(\operatorname{ATLAS} \gamma \gamma)
\end{array}\right.
$$

We use the combined Higgs mass $M_{h}^{\exp }=125.66 \pm 0.34 \mathrm{GeV}$ [92]. The $\chi^{2}$ definition in our fit is

$$
\chi^{2}=\sum_{i=1}^{22} \frac{\left(\mu_{i}-\mu_{i}^{\exp }\right)^{2}}{\sigma_{i}^{2}}+\frac{\left(M_{h}-M_{h}^{\exp }\right)^{2}}{\sigma_{M_{h}}^{2}} .
$$

where $\sigma_{i}$ and $\sigma_{M_{h}}$ only denote the experimental errors.

\subsection{The cross section of $b \bar{b} \rightarrow h h$ with SUSY-QCD correction}

We use CTEQ6L1 and CTEQ6m [93] for the leading order and SUSY-QCD calculation, respectively. The renormalization scale $\mu_{R}$ and factorization scale $\mu_{F}$ basically can vary between $M_{h} / 2$ and $2 M_{h}$. In order to compare our results with [44] where $\mu_{R}=\mu_{F}=M_{h} / 2$ is assumed, we also made this assumption in our calculation. The input parameters of the 


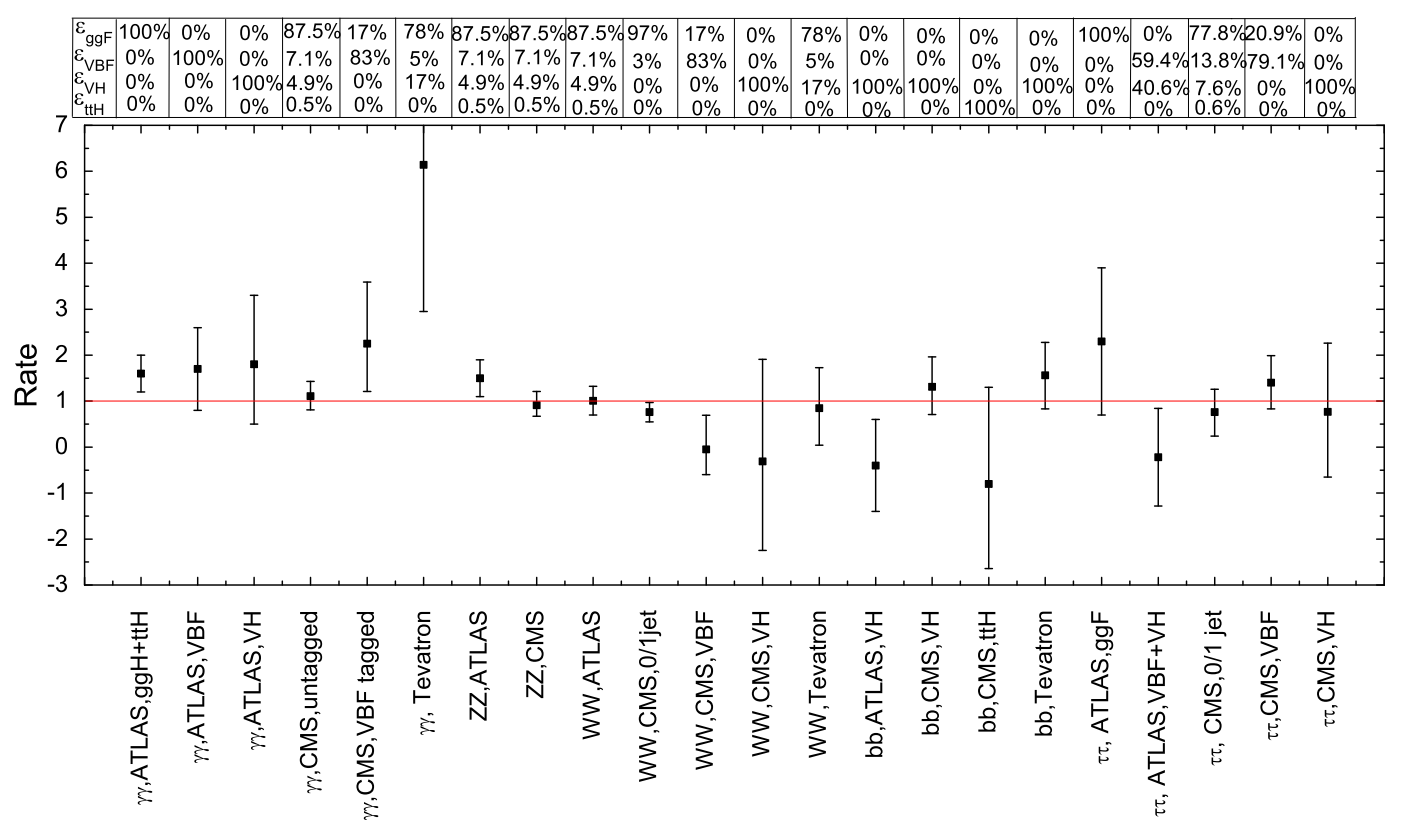

Figure 1. The measured signal strength of Higgs boson with their $1 \sigma$ error-bars and selection efficiencies $\epsilon_{p}$ for each production mode $p$ and decay mode at the $7+8 \mathrm{TeV}$ LHC and Tevatron.

SM are taken as [94]

$$
\begin{aligned}
& m_{b}=4.7 \mathrm{GeV}, \quad m_{t}=173.1 \mathrm{GeV}, \quad m_{Z}=91.19 \mathrm{GeV}, \\
& \sin ^{2} \theta_{W}=0.2228, \quad \alpha_{s}\left(m_{t}\right)=0.1033, \quad \alpha=1 / 128 .
\end{aligned}
$$

In figure 2, we display the parameter space satisfying the experimental constraints (i-iv), showing the cross sections of the SM-like Higgs pair productions via $b \bar{b}$ annihilation (with SUSY QCD correction) and gg fusion versus $M_{A}$ at the $14 \mathrm{TeV}$ LHC in MSSM and NMSSM. In this paper we aim to investigate the property of the $b \bar{b} \rightarrow h h$ production by including the SUSY QCD corrections. For the $g g \rightarrow h h$ production, we only calculate its cross section at one-loop level, not including the SUSY QCD corrections due to its small relative correction $[95,96]$ comparing the SUSY QCD correction on $b \bar{b} \rightarrow h h$ process. We used our own codes and combined them with Looptools to do our calculation. We checked our results with [27] and found good agreement.

We checked that our results agree with [44] for $b \bar{b} \rightarrow h h$ and with [41] for the gluon fusion process. We can see that due to the constraints from the LHC and B-physics, such as $H / A \rightarrow \tau^{+} \tau^{-}$[74] and $B_{s} \rightarrow \mu^{+} \mu^{-}$[97], the values of $m_{A}$ must be larger than about $300 \mathrm{GeV}$. In the MSSM the maximal cross section can still reach $50 \mathrm{fb}$ at $14 \mathrm{TeV}$ LHC, which can be competitive with $g g \rightarrow h h$. However, we also notice that the hadronic cross section proceeding through $b \bar{b} \rightarrow h h$ deceases when $m_{A}$ or $\tan \beta$ becomes large. The reason can be understood as follows. On the one hand, for a moderate $m_{A}$, the dominant contribution to $b \bar{b} \rightarrow h h$ comes from the resonant production $b \bar{b} \rightarrow H \rightarrow h h$. With the increase of $M_{A}$, the mass of $H$ gets heavy and then the production rate of $b \bar{b} \rightarrow h h$ is suppressed. Besides, 

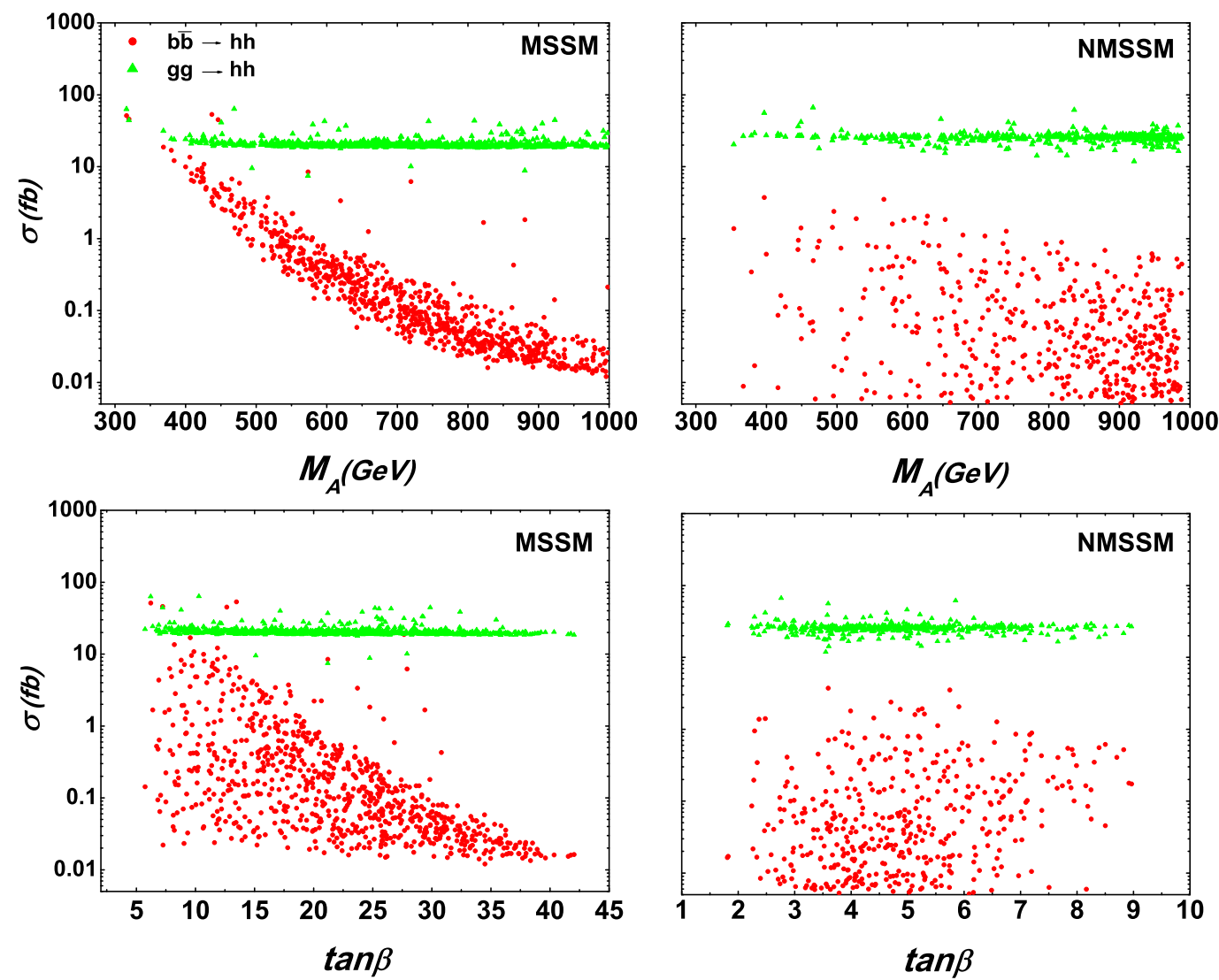

Figure 2. The scatter plot of the parameter space satisfying the experimental constraints (i-iv), showing the hadronic cross sections of the SM-like Higgs pair productions via $b \bar{b}$ annihilation (with SUSY QCD correction) and $g g$ fusion versus $M_{A}$ and $\tan \beta$ at the $14 \mathrm{TeV}$ LHC in MSSM and NMSSM.

the coupling of $h h H$ will approach to zero for a large $m_{A}$ and also leads to the reduction of the cross section. On the other hand, for a small $\tan \beta, H$ has a large branching ratio into a pair of Higgses $h h$ [98], for a large $\tan \beta$, the production rate of $b \bar{b} \rightarrow H$ can be enhanced but the branch ratio of $H \rightarrow h h$ is highly suppressed. So the total production rate of $b \bar{b} \rightarrow h h$ will become small.The decoupling behavior of the cross section proceeding through $g g \rightarrow h h$ can be understood with the following considerations: to predict a 125 Gev Higgs boson, a large $A_{t}$ is required, which induces a sizable SUSY effect for the process $g g \rightarrow h h . M_{A}$ affects the process $g g \rightarrow h h$ mainly through the Higgs mass $m_{h}$. So when we require $m_{h}$ in the range of $123-127 \mathrm{GeV}$, the process $g g \rightarrow h h$ is not sensitive to $M_{A}$. Further, since $g g \rightarrow h h$ is dominated by the stop loops, the value of $\tan \beta$ affects this process through the coupling $h \tilde{t}_{i} \tilde{t}_{j}$. Because this coupling is not sensitive to $\tan \beta$ for our surviving points, our results depend weakly on $\tan \beta$.

In NMSSM the SM-like Higgs boson $h$ with mass around $125 \mathrm{GeV}$ can be either $h_{1}$ or $h_{2}$. However, we focus on the $h=h_{2}$ scenario that is more welcomed by the naturalness. From figure 2 we can see that the maximal cross section of $b \bar{b} \rightarrow h h$ can only reach about 

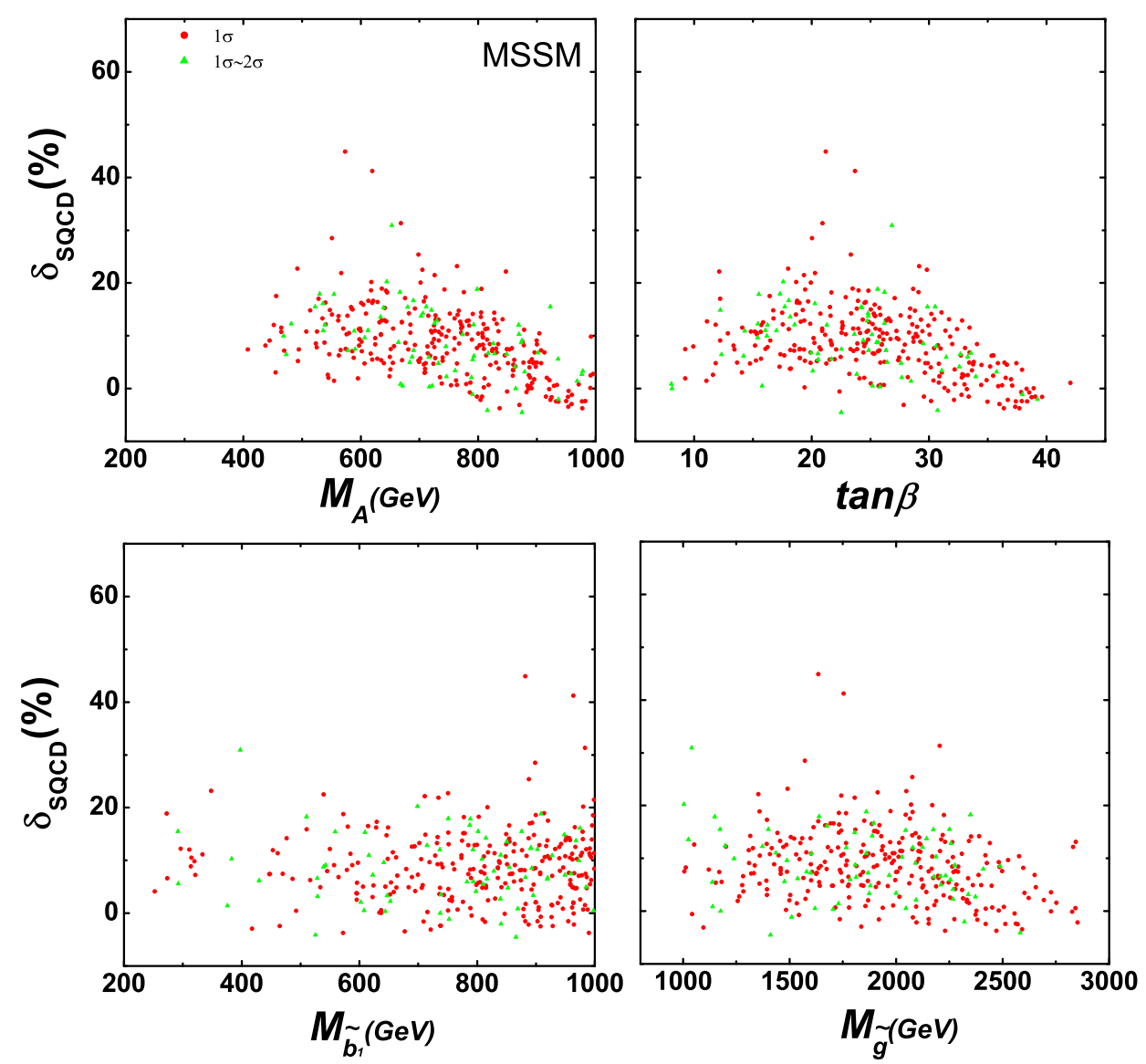

Figure 3. Same as figure 2, but showing the relative SUSY-QCD correction for the $b \bar{b} \rightarrow h h$ in the MSSM. Here the samples satisfying the experimental constraints (i-iv) are further classified according to the Higgs data: within $1 \sigma$ (red dots), outside $1 \sigma$ but within $2 \sigma$ (green triangles).

$4 \mathrm{fb}$, which is much smaller than $g g \rightarrow h h$. We find that the suppression of $b \bar{b} \rightarrow h h$ in NMSSM mainly has two reasons. One is that in NMSSM the $\tan \beta$ value is around 3-5 which is much smaller than in MSSM which is always larger than 10 . So the $\tan \beta$ enhancement on $h_{i} b \bar{b}$ coupling is not significant in NMSSM. The other reason is the $h_{3} h_{2} h_{2}$ coupling is suppressed for most surviving points (the main reason is the cancelation of different contributions). Besides, in the NMSSM the $125 \mathrm{GeV}$ Higgs mass requires a small $\tan \beta$ and a large $\lambda$. So the cross section of $b \bar{b} \rightarrow h h$ can hardly enhanced by $\tan \beta$.

To further investigate the influence of the Higgs data in figure 2 on the SUSY-QCD effect in $b \bar{b} \rightarrow h h$, we define the relative SUSY-QCD correction $\delta_{\mathrm{SQCD}}$ as

$$
\delta_{\mathrm{SQCD}}=\frac{\sigma_{\mathrm{SQCD}}-\sigma_{\mathrm{LO}}}{\sigma_{\mathrm{LO}}} .
$$

In our calculation we use the $\alpha_{s}^{\mathrm{LO}}$ for the LO cross-section and $\alpha_{s}^{\mathrm{NLO}}$ for the NLO crosssections, respectively. In figure 3 we show the dependence of $\delta_{\mathrm{SQCD}}$ for the $b \bar{b} \rightarrow h h$ on the SUSY parameters $M_{A}, \tan \beta$, the lightest sbottom mass $\left(m_{\tilde{b}_{1}}\right)$ and gluino mass $\left(m_{\tilde{g}}\right)$ in the MSSM. In this figure the samples satisfying the experimental constraints (i-iv) are further 

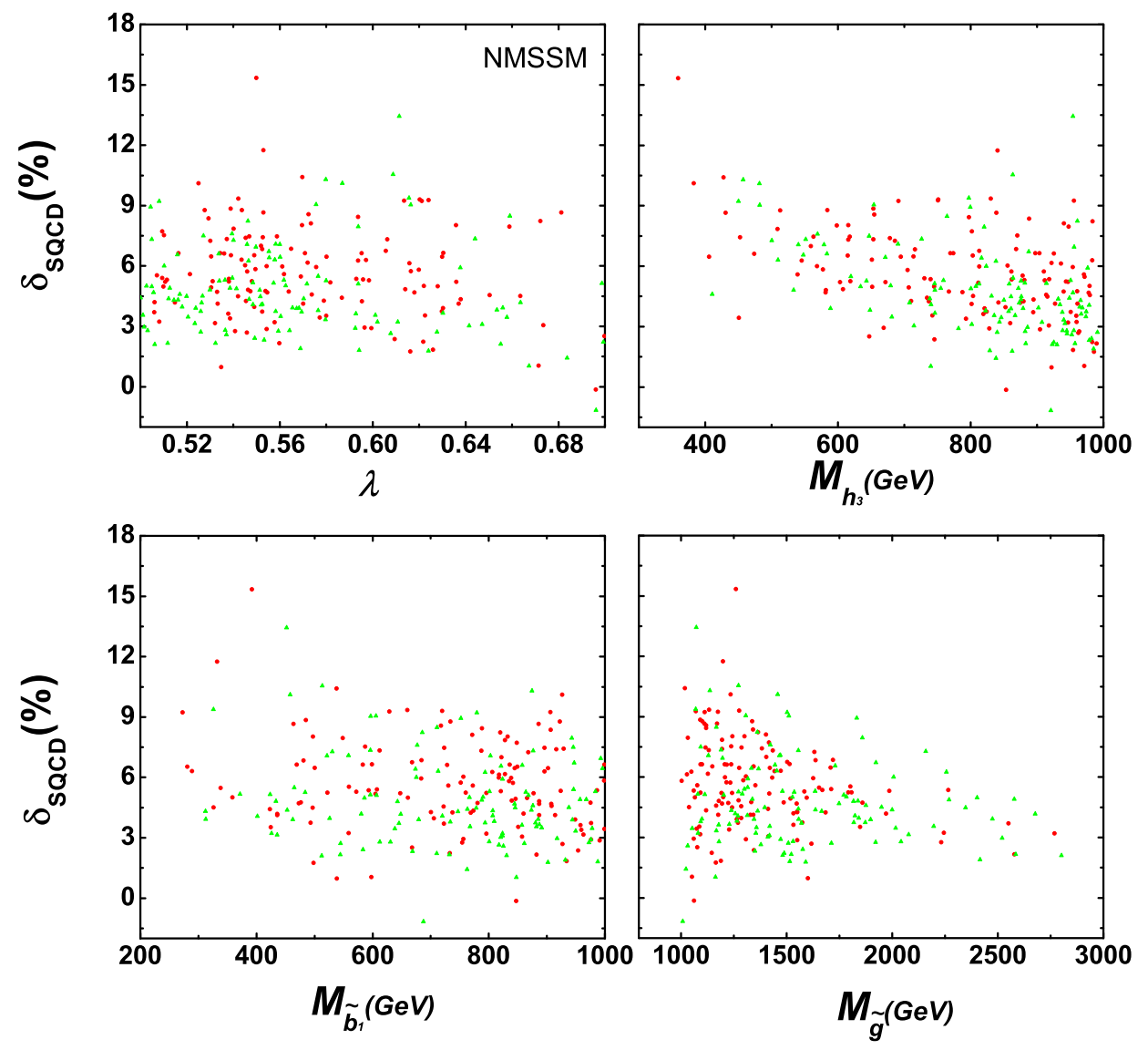

Figure 4. Same as figure 3, but for the NMSSM.

classified according to the Higgs data: we use the $\chi^{2}$ and the degree of freedom to calculate the p-value for each point and plot the points whose p-values are larger than $0.045(2 \sigma)$ and $0.318(1 \sigma)$. The degree of freedom is 15 [23(experimental observables)-8(free parameters)] for MSSM and 12 [23(experimental observables)-11(free parameters)] for NMSSM.From the upper panel we can see that a heavy $m_{A}(>400 \mathrm{GeV})$ and a moderate $\tan \beta(10 \sim 40)$ are favored by the Higgs data and the SUSY-QCD correction can maximally reach about $45 \%$ for the samples in $1 \sigma$ range. Similar to figure $2, \delta_{\mathrm{SQCD}}$ decreases when $m_{A}$ becomes heavy. From the lower panel we note that for heavy $m_{\tilde{b}_{1}}$ and $m_{\tilde{g}}$, the SUSY-QCD effects decouple slowly. This behavior is because that the SUSY-QCD corrections depend on the ratio of the SUSY parameters. For example, in the triangle diagrams, the SUSY-QCD correction to the vertex $h b \bar{b}$ is proportional to $M_{E W}^{2} / M_{A}^{2}$ and $M_{E W}^{2} / M_{\tilde{b}}^{2}[63,99-104]$. So only when all the sparticles and $m_{A}$ are heavy, the SUSY-QCD effect can completely decouple from the process of $b \bar{b} \rightarrow h h$.

The relative SUSY-QCD corrections for the $b \bar{b} \rightarrow h h$ in the NMSSM are presented in figure 4. It can be seen that the maximal SUSY-QCD correction can reach $15 \%$ for the samples in $1 \sigma$ range. From the upper panel we can see that $\delta_{S Q C D}$ becomes small with the increase of $\lambda$ or $m_{h_{3}}$. The reason is that with the increase of the $\lambda$, the $m_{h_{3}}$ gets heavy and 


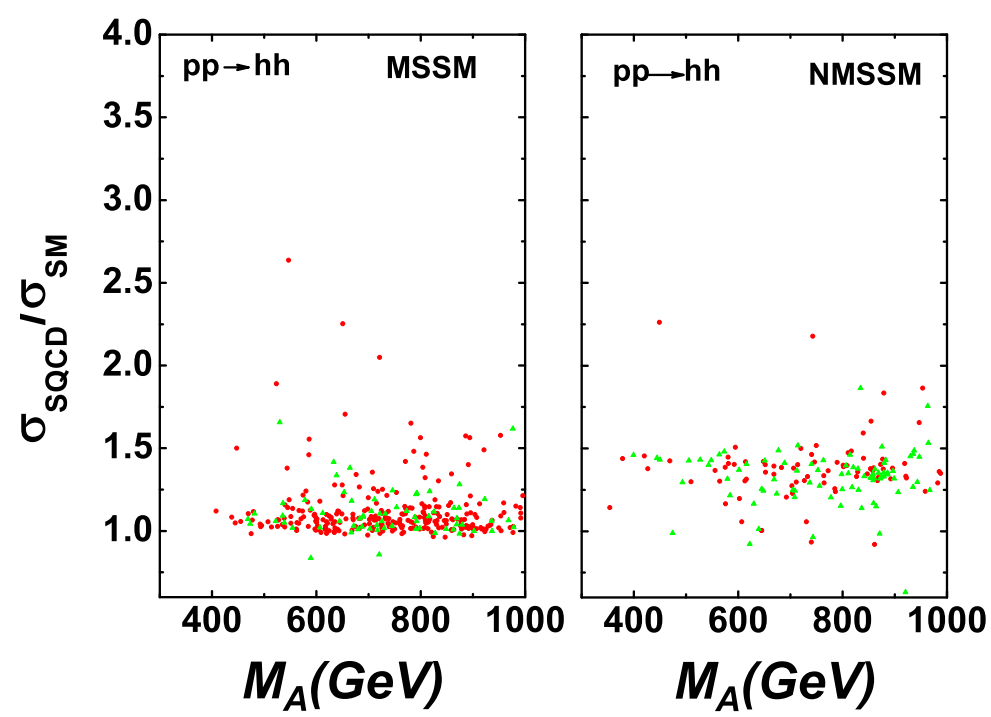

Figure 5. The total cross section of the Higgs pair production at the $14 \mathrm{TeV}$ LHC via both $b \bar{b}$ annihilation (include the SQCD correction) and $g g$ fusion (without the SQCD correction) in MSSM and NMSSM.

its contribution to the cross section becomes small. From the lower panel we see that, due to the residual effects of the sparticles, the SUSY-QCD corrections can still reach about $9 \%$ for heavy sbottom and gluino.

In figure 5 we show the total cross section of the Higgs pair production at the $14 \mathrm{TeV}$ LHC (via both $b \bar{b}$ annihilation and $g g$ fusion) for the samples in the $1 \sigma$ and $2 \sigma$ ranges of the Higgs data. We can see that in the $1 \sigma$ range the total cross section can be maximally enhanced by a factor of 2.7 and 2.2 in the MSSM and NMSSM, respectively.

Finally, considering the null results of the direct search for sparticles at the LHC, we investigate the SUSY-QCD effect in Higgs pair production in the limit of heavy sparticles. For simplicity, we assume a common mass $M_{S U S Y}$ for all relevant SUSY mass parameters: $M_{S U S Y}=M_{\tilde{Q}}=M_{\tilde{D}}=A_{t}=A_{b}=M_{\tilde{g}}=M_{\mu}$. In figure 6 we display the ratio of $\sigma_{\mathrm{SUSY}}^{p p \rightarrow h h} / \sigma_{\mathrm{SM}}^{p p \rightarrow h h}$. We can see that for $M_{S U S Y}=1 \mathrm{TeV}$, the ratios will maximally reach 3 and 2 in the MSSM and NMSSM, respectively. When $M_{S U S Y}$ goes up to $5 \mathrm{TeV}$, the enhancements become weak but can still reach 1.8 and 1.4 in the MSSM and NMSSM, respectively. So the effects of heavy sparticles decouple quite slowly from the Higgs pair production. We checked that the SUSY effects decouple quickly in $b \bar{b} \rightarrow h h$ but slowly in $g g \rightarrow h h$.

\section{Conclusion}

We considered the current experimental constraints on the parameter space of the MSSM and NMSSM. Then in the allowed parameter space we examined $b \bar{b} \rightarrow h h(h$ is the $125 \mathrm{GeV}$ SM-like Higg boson) with one-loop SUSY QCD correction and compared it with $g g \rightarrow h h$. 

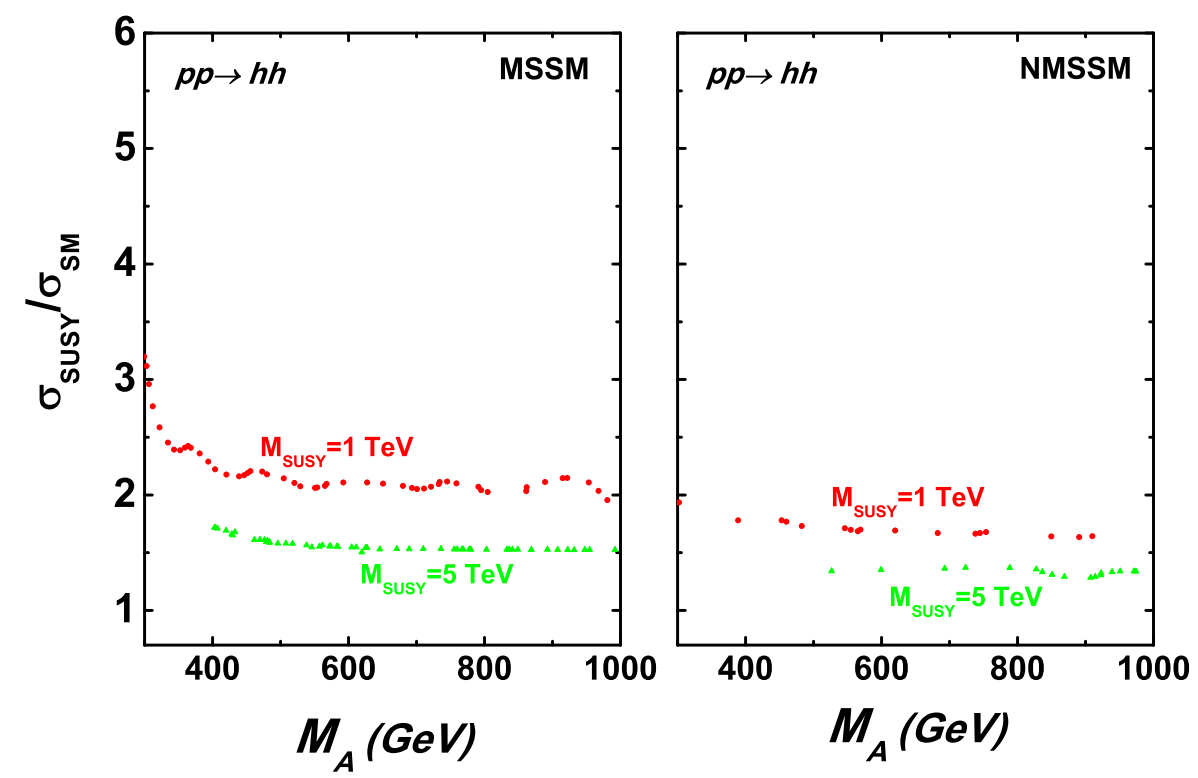

Figure 6. The cross section of Higgs pair production via both $b \bar{b}$ annihilation (include the SQCD correction) and $g g$ fusion (without the SQCD correction) in MSSM and NMSSM for heavy sparticle masses at $14 \mathrm{TeV}$ LHC.

We obtained the following observations: (i) For the MSSM the production rate of $b \bar{b} \rightarrow h h$ (with one-loop SUSY QCD correction) can reach $50 \mathrm{fb}$ and thus can be competitive with $g g \rightarrow h h$, while for the NMSSM $b \bar{b} \rightarrow h h$ has a much smaller rate than $g g \rightarrow h h$ due to the suppression of the $h b \bar{b}$ coupling; (ii) The SUSY-QCD correction to $b \bar{b} \rightarrow h h$ is sizable, which can reach $45 \%$ for the MSSM and $15 \%$ for the NMSSM within the $1 \sigma$ region of the Higgs data; (iii) In the heavy SUSY limit (all soft mass parameters become heavy), the SUSY effects decouple rather slowly from the Higgs pair production, which, for $M_{\mathrm{SUSY}}=5 \mathrm{TeV}$ and $m_{A}<1 \mathrm{TeV}$, can enhance the production rate by a factor of 1.5 and 1.3 for the MSSM and NMSSM, respectively. Therefore, the Higgs pair production may be helpful for unraveling the effects of heavy SUSY.

\section{Acknowledgments}

We appreciate the helpful discussions with Junjie Cao, Ning Liu, Wenyu Wang and Yang Zhang. This work was supported in part by the ARC Centre of Excellence for Particle Physics at the Tera-scale, by the National Natural Science Foundation of China (NNSFC) under grant No. 10775039, 11075045, 11275245, 10821504 and 11135003, by Ri-Xin Foundation of BJUT from China and by the Startup Foundation for Doctors of Henan Normal University under contract No.11112.

Open Access. This article is distributed under the terms of the Creative Commons Attribution License (CC-BY 4.0), which permits any use, distribution and reproduction in any medium, provided the original author(s) and source are credited. 


\section{References}

[1] ATLAS collaboration, An update of combined measurements of the new Higgs-like boson with high mass resolution channels, ATLAS-CONF-2012-170 (2012).

[2] CMS collaboration, Combination of standard model Higgs boson searches and measurements of the properties of the new boson with a mass near 125 GeV, CMS-PAS-HIG-12-045 (2012).

[3] J. Ellis and T. You, Global Analysis of Experimental Constraints on a Possible Higgs-Like Particle with Mass $125 \mathrm{GeV}$, JHEP 06 (2012) 140 [arXiv:1204.0464] [INSPIRE].

[4] J. Ellis and T. You, Global Analysis of the Higgs Candidate with Mass 125 GeV, JHEP 09 (2012) 123 [arXiv:1207.1693] [INSPIRE].

[5] J. Ellis and K.A. Olive, Revisiting the Higgs Mass and Dark Matter in the CMSSM, Eur. Phys. J. C 72 (2012) 2005 [arXiv: 1202.3262] [INSPIRE].

[6] U. Ellwanger and C. Hugonie, Higgs bosons near $125 \mathrm{GeV}$ in the NMSSM with constraints at the GUT scale, Adv. High Energy Phys. 2012 (2012) 625389 [arXiv:1203.5048].

[7] R. Benbrik et al., Confronting the MSSM and the NMSSM with the Discovery of a Signal in the two Photon Channel at the LHC, Eur. Phys. J. C 72 (2012) 2171 [arXiv:1207.1096] [INSPIRE].

[8] M. Carena, S. Gori, N.R. Shah and C.E. Wagner, A 125 GeV SM-like Higgs in the MSSM and the $\gamma \gamma$ rate, JHEP 03 (2012) 014 [arXiv:1112.3336] [INSPIRE].

[9] M. Carena, S. Heinemeyer, O. Stål, C. Wagner and G. Weiglein, MSSM Higgs Boson Searches at the LHC: Benchmark Scenarios after the Discovery of a Higgs-like Particle, Eur. Phys. J. C 73 (2013) 2552 [arXiv:1302.7033] [INSPIRE].

[10] J. Cao, Z. Heng, D. Li and J.M. Yang, Current experimental constraints on the lightest Higgs boson mass in the constrained MSSM, Phys. Lett. B 710 (2012) 665 [arXiv:1112.4391] [INSPIRE].

[11] J.-J. Cao, Z.-X. Heng, J.M. Yang, Y.-M. Zhang and J.-Y. Zhu, A SM-like Higgs near 125 GeV in low energy SUSY: a comparative study for MSSM and NMSSM, JHEP 03 (2012) 086 [arXiv:1202.5821] [INSPIRE].

[12] J. Cao, Z. Heng, J.M. Yang and J. Zhu, Status of low energy SUSY models confronted with the LHC 125 GeV Higgs data, JHEP 10 (2012) 079 [arXiv:1207.3698] [INSPIRE].

[13] J. Cao, L. Wu, P. Wu and J.M. Yang, The Z+photon and diphoton decays of the Higgs boson as a joint probe of low energy SUSY models, JHEP 09 (2013) 043 [arXiv:1301.4641] [INSPIRE].

[14] A. Djouadi, W. Kilian, M. Muhlleitner and P. Zerwas, Production of neutral Higgs boson pairs at LHC, Eur. Phys. J. C 10 (1999) 45 [hep-ph/9904287] [INSPIRE].

[15] U. Baur, T. Plehn and D.L. Rainwater, Probing the Higgs selfcoupling at hadron colliders using rare decays, Phys. Rev. D 69 (2004) 053004 [hep-ph/0310056] [INSPIRE].

[16] J.M. Butterworth, A.R. Davison, M. Rubin and G.P. Salam, Jet substructure as a new Higgs search channel at the LHC, Phys. Rev. Lett. 100 (2008) 242001 [arXiv:0802.2470] [INSPIRE].

[17] J. Baglio et al., The measurement of the Higgs self-coupling at the LHC: theoretical status, JHEP 04 (2013) 151 [arXiv:1212.5581] [INSPIRE]. 
[18] M.J. Dolan, C. Englert and M. Spannowsky, Higgs self-coupling measurements at the LHC, JHEP 10 (2012) 112 [arXiv:1206.5001] [INSPIRE].

[19] M.J. Dolan, C. Englert and M. Spannowsky, New Physics in LHC Higgs boson pair production, Phys. Rev. D 87 (2013) 055002 [arXiv:1210.8166] [INSPIRE].

[20] A. Papaefstathiou, L.L. Yang and J. Zurita, Higgs boson pair production at the LHC in the $b \bar{b} W^{+} W^{-}$channel, Phys. Rev. D 87 (2013) 011301 [arXiv: 1209.1489] [InSPIRE].

[21] D.A. Dicus, C. Kao and S.S. Willenbrock, Higgs Boson Pair Production From Gluon Fusion, Phys. Lett. B 203 (1988) 457 [INSPIRE].

[22] S. Dawson, S. Dittmaier and M. Spira, Neutral Higgs boson pair production at hadron colliders: QCD corrections, Phys. Rev. D 58 (1998) 115012 [hep-ph/9805244] [INSPIRE].

[23] D.Y. Shao, C.S. Li, H.T. Li and J. Wang, Threshold resummation effects in Higgs boson pair production at the LHC, JHEP 07 (2013) 169 [arXiv:1301.1245] [INSPIRE].

[24] D. de Florian and J. Mazzitelli, Two-loop virtual corrections to Higgs pair production, Phys. Lett. B 724 (2013) 306 [arXiv:1305.5206] [INSPIRE].

[25] J. Grigo, J. Hoff, K. Melnikov and M. Steinhauser, On the Higgs boson pair production at the LHC, Nucl. Phys. B 875 (2013) 1 [arXiv:1305.7340] [INSPIRE].

[26] T. Plehn, M. Spira and P. Zerwas, Pair production of neutral Higgs particles in gluon-gluon collisions, Nucl. Phys. B 479 (1996) 46 [Erratum ibid. B 531 (1998) 655] [hep-ph/9603205] [INSPIRE].

[27] H. Sun, Y.-J. Zhou and H. Chen, Constraints on large-extra-dimensions model through 125 GeV Higgs pair production at the LHC, Eur. Phys. J. 72 (2012) 2011 [arXiv:1211.5197] [INSPIRE].

[28] H. Sun and Y.-J. Zhou, Enhancement of Loop induced 125GeV Higgs pair production through Large-Extra-Dimensions model at the LHC, JHEP 11 (2012) 127 [arXiv:1211.6201] [INSPIRE].

[29] R. Grober and M. Muhlleitner, Composite Higgs Boson Pair Production at the LHC, JHEP 06 (2011) 020 [arXiv: 1012.1562] [INSPIRE].

[30] M. Gouzevitch et al., Scale-invariant resonance tagging in multijet events and new physics in Higgs pair production, JHEP 07 (2013) 148 [arXiv:1303.6636] [INSPIRE].

[31] E. Asakawa, D. Harada, S. Kanemura, Y. Okada and K. Tsumura, Higgs boson pair production in new physics models at hadron, lepton and photon colliders, Phys. Rev. D 82 (2010) 115002 [arXiv: 1009.4670] [InSPIRE].

[32] W. Ma, C.-X. Yue and Y.-Z. Wang, Pair production of neutral Higgs bosons from the left-right twin Higgs model at the ILC and LHC, Phys. Rev. D 79 (2009) 095010 [arXiv:0905.0597] [INSPIRE].

[33] X.-F. Han, L. Wang and J.M. Yang, Higgs-pair Production and Decay in Simplest Little Higgs Model, Nucl. Phys. B 825 (2010) 222 [arXiv:0908.1827] [INSPIRE].

[34] L. Wang and X.-F. Han, Standard-model-like Higgs-pair production and decay in left-right twin Higgs model, Phys. Lett. B 696 (2011) 79 [INSPIRE].

[35] M. Moretti, S. Moretti, F. Piccinini, R. Pittau and J. Rathsman, Production of Light Higgs Pairs in 2-Higgs Doublet Models via the Higgs-strahlung Process at the LHC, JHEP 11 (2010) 097 [arXiv: 1008.0820] [INSPIRE]. 
[36] N.D. Christensen, T. Han and T. Li, Pair Production of MSSM Higgs Bosons in the Non-decoupling Region at the LHC, Phys. Rev. D 86 (2012) 074003 [arXiv:1206.5816] [INSPIRE].

[37] F. Maltoni, Z. Sullivan and S. Willenbrock, Higgs-boson production via bottom-quark fusion, Phys. Rev. D 67 (2003) 093005 [hep-ph/0301033] [INSPIRE].

[38] R.V. Harlander and W.B. Kilgore, Higgs boson production in bottom quark fusion at next-to-next-to leading order, Phys. Rev. D 68 (2003) 013001 [hep-ph/0304035] [INSPIRE].

[39] S. Dittmaier, M. Kramer, A. Muck and T. Schluter, MSSM Higgs-boson production in bottom-quark fusion: Electroweak radiative corrections, JHEP 03 (2007) 114 [hep-ph/0611353] [INSPIRE].

[40] A. Barrientos Bendezu and B.A. Kniehl, Pair production of neutral Higgs bosons at the CERN large hadron collider, Phys. Rev. D 64 (2001) 035006 [hep-ph/0103018] [InSPIRE].

[41] J. Cao, Z. Heng, L. Shang, P. Wan and J.M. Yang, Pair Production of a 125 GeV Higgs Boson in MSSM and NMSSM at the LHC, JHEP 04 (2013) 134 [arXiv:1301.6437] [INSPIRE].

[42] U. Ellwanger, Higgs pair production in the NMSSM at the LHC, JHEP 08 (2013) 077 [arXiv: 1306.5541] [INSPIRE].

[43] ATLAS collaboration, Search for direct third generation squark pair production in final states with missing transverse momentum and two b-jets in $\sqrt{s}=8 \mathrm{TeV}$ pp collisions with the ATLAS detector, ATLAS-CONF-2013-053 (2013).

[44] S. Dawson, C. Kao and Y. Wang, SUSY QCD Corrections to Higgs Pair Production from Bottom Quark Fusion, Phys. Rev. D 77 (2008) 113005 [arXiv:0710.4331] [INSPIRE].

[45] C. Brust, A. Katz, S. Lawrence and R. Sundrum, SUSY, the Third Generation and the LHC, JHEP 03 (2012) 103 [arXiv:1110.6670] [InSPIRE].

[46] J.L. Feng and D. Sanford, A Natural 125 GeV Higgs Boson in the MSSM from Focus Point Supersymmetry with A-Terms, Phys. Rev. D 86 (2012) 055015 [arXiv:1205.2372] [INSPIRE].

[47] B. Allanach and B. Gripaios, Hide and Seek With Natural Supersymmetry at the LHC, JHEP 05 (2012) 062 [arXiv:1202.6616] [INSPIRE].

[48] S. Akula, M. Liu, P. Nath and G. Peim, Naturalness, Supersymmetry and Implications for LHC and Dark Matter, Phys. Lett. B 709 (2012) 192 [arXiv:1111.4589] [INSPIRE].

[49] L.J. Hall, D. Pinner and J.T. Ruderman, A Natural SUSY Higgs Near 126 GeV, JHEP 04 (2012) 131 [arXiv:1112.2703] [INSPIRE].

[50] H. Baer, V. Barger, P. Huang and X. Tata, Natural Supersymmetry: LHC, dark matter and ILC searches, JHEP 05 (2012) 109 [arXiv:1203.5539] [INSPIRE].

[51] M. Papucci, J.T. Ruderman and A. Weiler, Natural SUSY Endures, JHEP 09 (2012) 035 [arXiv:1110.6926] [INSPIRE].

[52] J. Cao, C. Han, L. Wu, J.M. Yang and Y. Zhang, Probing Natural SUSY from Stop Pair Production at the LHC, JHEP 11 (2012) 039 [arXiv:1206.3865] [INSPIRE].

[53] J. Rosiek, Complete set of Feynman rules for the MSSM: Erratum, hep-ph/9511250 [INSPIRE].

[54] F. Franke and H. Fraas, Neutralinos and Higgs bosons in the next-to-minimal supersymmetric standard model, Int. J. Mod. Phys. A 12 (1997) 479 [hep-ph/9512366]. 
[55] M. Aivazis, J.C. Collins, F.I. Olness and W.-K. Tung, Leptoproduction of heavy quarks. 2. A Unified QCD formulation of charged and neutral current processes from fixed target to collider energies, Phys. Rev. D 50 (1994) 3102 [hep-ph/9312319] [InSPIRE].

[56] J.C. Collins, Hard scattering factorization with heavy quarks: a general treatment, Phys. Rev. D 58 (1998) 094002 [hep-ph/9806259] [INSPIRE].

[57] M. Kramer, F.I. Olness and D.E. Soper, Treatment of heavy quarks in deeply inelastic scattering, Phys. Rev. D 62 (2000) 096007 [hep-ph/0003035] [INSPIRE].

[58] T.N. Dao, W. Hollik and D.N. Le, $W^{\mp} H^{ \pm}$production and CP asymmetry at the LHC, Phys. Rev. D 83 (2011) 075003 [arXiv: 1011.4820] [INSPIRE].

[59] R. Enberg, R. Pasechnik and O. Stal, Enhancement of associated $H^{ \pm} W^{\mp}$ production in the NMSSM, Phys. Rev. D 85 (2012) 075016 [arXiv:1112.4699] [InSPIRE].

[60] D.M. Pierce, J.A. Bagger, K.T. Matchev and R.-j. Zhang, Precision corrections in the minimal supersymmetric standard model, Nucl. Phys. B 491 (1997) 3 [hep-ph/9606211] [INSPIRE].

[61] M.S. Carena, D. Garcia, U. Nierste and C.E. Wagner, Effective Lagrangian for the $\bar{t} b H^{+}$ interaction in the MSSM and charged Higgs phenomenology, Nucl. Phys. B 577 (2000) 88 [hep-ph/9912516] [INSPIRE].

[62] M.S. Carena, J.R. Ellis, S. Mrenna, A. Pilaftsis and C. Wagner, Collider probes of the MSSM Higgs sector with explicit CP-violation, Nucl. Phys. B 659 (2003) 145 [hep-ph/0211467] [INSPIRE].

[63] J. Guasch, P. Hafliger and M. Spira, MSSM Higgs decays to bottom quark pairs revisited, Phys. Rev. D 68 (2003) 115001 [hep-ph/0305101] [INSPIRE].

[64] D. Noth and M. Spira, Higgs Boson Couplings to Bottom Quarks: Two-Loop Supersymmetry-QCD Corrections, Phys. Rev. Lett. 101 (2008) 181801 [arXiv:0808.0087] [INSPIRE].

[65] D. Noth and M. Spira, Supersymmetric Higgs Yukawa Couplings to Bottom Quarks at next-to-next-to-leading Order, JHEP 06 (2011) 084 [arXiv:1001.1935] [INSPIRE].

[66] L. Mihaila and C. Reisser, $O\left(\alpha_{s}^{2}\right)$ corrections to fermionic Higgs decays in the MSSM, JHEP 08 (2010) 021 [arXiv: 1007.0693] [INSPIRE].

[67] J. Baglio et al., NMSSMCALC: A Program Package for the Calculation of Loop-Corrected Higgs Boson Masses and Decay Widths in the (Complex) NMSSM, arXiv:1312.4788 [INSPIRE].

[68] E. Braaten and J. Leveille, Higgs Boson Decay and the Running Mass, Phys. Rev. D 22 (1980) 715 [inSPIRE].

[69] J. Gunion and H.E. Haber, Higgs Bosons in Supersymmetric Models. 1., Nucl. Phys. B 272 (1986) 1 [Erratum ibid. B 402 (1993) 567] [InSPIRE].

[70] U. Ellwanger, J.F. Gunion and C. Hugonie, NMHDECAY: A Fortran Code for the Higgs Masses, Couplings and Decay Widths in the NMSSM, JHEP 02 (2005) 066 [hep-ph/0406215] [INSPIRE].

[71] U. Ellwanger and C. Hugonie, NMHDECAY 2.0: An updated program for Sparticle masses, Higgs masses, couplings and decay widths in the NMSSM, Comput. Phys. Commun. 175 (2006) 290 [hep-ph/0508022] [INSPIRE]. 
[72] G. Bélanger, F. Boudjema, C. Hugonie, A. Pukhov and A. Semenov, Relic density of dark matter in the NMSSM, JCAP 09 (2005) 001 [hep-ph/0505142] [INSPIRE].

[73] T. Hahn and M. Pérez-Victoria, Automatized one loop calculations in four-dimensions and D-dimensions, Comput. Phys. Commun. 118 (1999) 153 [hep-ph/9807565] [InSPIRE].

[74] CMS collaboration, Higgs to tau tau (MSSM) (HCP), CMS-PAS-HIG-12-050 (2012).

[75] ATLAS collaboration, Search for charged Higgs bosons decaying via H+- $\mathrm{i}$ tau nu in ttbar events using $4.6 \mathrm{fb} 1$ of $p p$ collision data at $\sqrt{s}=7 \mathrm{TeV}$ with the ATLAS detector, ATLAS-CONF-2012-011 (2012).

[76] G. Altarelli and R. Barbieri, Vacuum polarization effects of new physics on electroweak processes, Phys. Lett. B 253 (1991) 161 [InSPIRE].

[77] M.E. Peskin and T. Takeuchi, Estimation of oblique electroweak corrections, Phys. Rev. D 46 (1992) 381 [INSPIRE].

[78] XENON100 collaboration, E. Aprile et al., Dark Matter Results from 100 Live Days of XENON100 Data, Phys. Rev. Lett. 107 (2011) 131302 [arXiv:1104.2549] [INSPIRE].

[79] M. Davier, A. Hoecker, B. Malaescu, C. Yuan and Z. Zhang, Reevaluation of the hadronic contribution to the muon magnetic anomaly using new $e^{+} e^{-} \rightarrow \pi^{+} \pi^{-}$cross section data from BABAR, Eur. Phys. J. C 66 (2010) 1 [arXiv:0908.4300] [inSPIRE].

[80] ATLAS collaboration, Search for squarks and gluinos with the ATLAS detector in final states with jets and missing transverse momentum and $20.3 \mathrm{fb}^{-1}$ of $\sqrt{\mathrm{s}}=8 \mathrm{TeV}$ proton-proton collision data, ATLAS-CONF-2013-047 (2013).

[81] ATLAS collaboration, Search for squarks and gluinos in events with isolated leptons, jets and missing transverse momentum at $\sqrt{s}=8 \mathrm{TeV}$ with the ATLAS detector, ATLAS-CONF-2013-062 (2013).

[82] ATLAS collaboration, Search for strongly produced supersymmetric particles in decays with two leptons at $\sqrt{s}=8 \mathrm{TeV}$, ATLAS-CONF-2013-089 (2013).

[83] ATLAS collaboration, Search for direct-slepton and direct-chargino production in final states with two opposite-sign leptons, missing transverse momentum and no jets in 20/fb of pp collisions at $\sqrt{s}=8 \mathrm{TeV}$ with the ATLAS detector, ATLAS-CONF-2013-049 (2013).

[84] ATLAS collaboration, Searches for direct scalar top pair production in final states with two leptons using the stransverse mass variable and a multivariate analysis technique in $\sqrt{s}=8 \mathrm{TeV}$ pp collisions using 20.3 fb-1 of ATLAS data, ATLAS-CONF-2013-065 (2013).

[85] ATLAS collaboration, Search for direct top squark pair production in final states with one isolated lepton, jets and missing transverse momentum in sqrts $=8 \mathrm{TeV}$ pp collisions using $21 \mathrm{fb}^{-1}$ of ATLAS data, ATLAS-CONF-2013-037 (2013).

[86] ATLAS collaboration, Search for direct production of the top squark in the all-hadronic ttbar + etmiss final state in $21 \mathrm{fb}-1$ of p-pcollisions at $\sqrt{s}=8 \mathrm{TeV}$ with the ATLAS detector, ATLAS-CONF-2013-024 (2013).

[87] ATLAS collaboration, Search for strongly produced superpartners in final states with two same sign leptons with the ATLAS detector using $21 \mathrm{fb}-1$ of proton-proton collisions at $\sqrt{s}=8 \mathrm{TeV}$., ATLAS-CONF-2013-007 (2013).

[88] ATLAS collaboration, Search for direct third-generation squark pair production in final states with missing transverse momentum and two b-jets in $\sqrt{s}=8 \mathrm{TeV} p p$ collisions with the ATLAS detector, JHEP 10 (2013) 189 [arXiv:1308.2631] [INSPIRE]. 
[89] K. Cheung, J.S. Lee and P.-Y. Tseng, Higgs Precision (Higgcision) Era begins, JHEP 05 (2013) 134 [arXiv: 1302.3794] [INSPIRE].

[90] CMS collaboration, Combination of standard model Higgs boson searches and measurements of the properties of the new boson with a mass near $125 \mathrm{GeV}$, CMS-PAS-HIG-13-005 (2013).

[91] ATLAS collaboration, Combined measurements of the mass and signal strength of the Higgs-like boson with the ATLAS detector using up to $25 \mathrm{fb}^{-1}$ of proton-proton collision data, ATLAS-CONF-2013-014 (2013).

[92] P.P. Giardino, K. Kannike, I. Masina, M. Raidal and A. Strumia, The universal Higgs fit, arXiv: 1303.3570 [INSPIRE].

[93] J. Pumplin et al., New generation of parton distributions with uncertainties from global QCD analysis, JHEP 07 (2002) 012 [hep-ph/0201195] [INSPIRE].

[94] Particle Data Group collaboration, J. Beringer et al., Review of Particle Physics (RPP), Phys. Rev. D 86 (2012) 010001 [InSPIRE].

[95] T. Plehn, M. Spira and P. Zerwas, Pair production of neutral Higgs particles in gluon-gluon collisions, Nucl. Phys. B 479 (1996) 46 [Erratum ibid. B 531 (1998) 655] [hep-ph/9603205] [INSPIRE].

[96] S. Dawson, S. Dittmaier and M. Spira, Neutral Higgs boson pair production at hadron colliders: QCD corrections, Phys. Rev. D 58 (1998) 115012 [hep-ph/9805244] [INSPIRE].

[97] LHCB collaboration, First Evidence for the Decay $B_{s}^{0} \rightarrow \mu^{+} \mu^{-}$, Phys. Rev. Lett. 110 (2013) 021801 [arXiv:1211.2674] [INSPIRE].

[98] M.J. Dolan, C. Englert and M. Spannowsky, New Physics in LHC Higgs boson pair production, Phys. Rev. D 87 (2013) 055002 [arXiv:1210.8166] [INSPIRE].

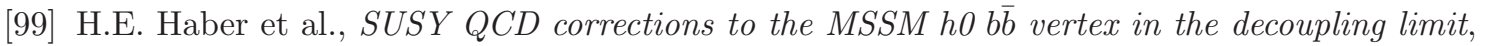
Phys. Rev. D 63 (2001) 055004 [hep-ph/0007006] [INSPIRE].

[100] G.-p. Gao, G.-r. Lu, Z.-h. Xiong and J.M. Yang, Loop effects and nondecoupling property of SUSY QCD in $g b \rightarrow t H^{-}$, Phys. Rev. D 66 (2002) 015007 [hep-ph/0202016] [INSPIRE].

[101] G. Gao, R.J. Oakes and J.M. Yang, Heavy supersymmetric particle effects in Higgs boson production associated with a bottom quark pair at LHC, Phys. Rev. D 71 (2005) 095005 [hep-ph/0412356] [INSPIRE].

[102] J.-j. Cao, G.-p. Gao, R.J. Oakes and J.M. Yang, Higgs-boson production associated with a bottom quark at hadron colliders with supersymmetric QCD corrections, Phys. Rev. D 68 (2003) 075012 [hep-ph/0210211] [INSPIRE].

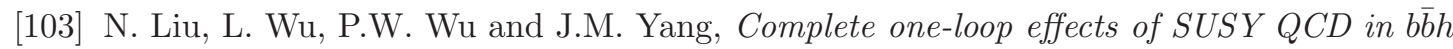
production at the LHC under current experimental constraints, JHEP 01 (2013) 161 [arXiv: 1208.3413] [INSPIRE].

[104] W. Wang, Z. Xiong and J.M. Yang, Residual effects of heavy sparticles in bottom quark Yukawa coupling: A Comparative study for MSSM and NMSSM,

Phys. Lett. B 680 (2009) 167 [arXiv:0901.3818] [INSPIRE]. 